\title{
A new species of Leuctridae discovered by means of molecular and biochemical approaches: Tyrrhenoleuctra antoninoi n. sp. (Insecta: Plecoptera)
}

\author{
ROMOLO FOCHETTI ${ }^{1} \&$ JOSÉ MANUEL TIERNO DE FIGUEROA $^{2}$ \\ ${ }^{\prime}$ Dipartimento di Scienze Ambientali. Università degli Studi della Tuscia. Largo dell'Università, 01100 - Viterbo, Italy. \\ E-mail: fochetti@unitus.it \\ ${ }^{2}$ Departamento de Biología Animal. Facultad de Ciencias. Universidad de Granada. 18071. Granada. España. E-mail: jmtdef@ ugr.es
}

\begin{abstract}
A new species of Tyrrhenoleuctra from the Balearic Islands, T. antoninoi sp. n., is described on the basis of molecular and biochemical characters. Recent biochemical and molecular analysis clearly demonstrates the distinctness of this species. We describe its relatedness to other species in the genus. We also refer all specimens from the Balearic Islands previously assigned to T. minuta to T. antoninoi. As with all Tyrrhenoleuctra species, T. antoninoi shows marked phenotypic plasticity, presumably due to the variability inherent in temporary streams in which it inhabits.
\end{abstract}

Key words: Tyrrhenoleuctra antoninoi, Plecoptera, Balearic Islands, temporary streams, phenotypic plasticity

\section{Introduction}

The genus Tyrrhenoleuctra shows a western Mediterranean distribution and presently includes three species: T. minuta (Klapálek, 1901), T. tangerina (Navás, 1922) and T. zavattarii (Consiglio, 1956). They occur mainly in temporary freshwater streams, sometimes also in lowland streams at sea level, a very unusual habitat for Plecoptera. The first two species have been recorded from the southern Iberian Peninsula and northern Africa, while T. zavattarii is distributed in Corsica and Sardinia. Tyrrhenoleuctra has been collected in the Balearic Islands (Pons 1986; García Avilés 1990; Tierno de Figueroa et al. 2003; Zwick in litteris), but, due to the lack of reliable morphological characters, it has not been determined at the species level (Sánchez-Ortega \& Tierno 1996).

The species of Tyrrhenoleuctra have traditionally been described and identified on the basis of genitalia morphology and adult body size (Klapálek 1901, Navás 1922, Consiglio 1956, Aubert 1963). However, these descriptions were based on only a few individuals from allopatric populations. Recent analysis from a wide geographic range, show extensive intra- and interspecific variation in genital characters (Sezzi et al. 2000, Sezzi 2001, Tierno de Figueroa \& Fochetti 2001). Thus, morphological characters can not be confidently and consistently used for identification. Also, purely morphological description of new taxa in the genus is severely inhibited by this high degree of variation (Fochetti et al. 2008).

Fochetti et al. (2004, 2008), Sezzi et al. (2000), Sezzi (2001) studied the biochemical and molecular genetic variation of Tyrrhenoleuctra across the entire geographic range of the genus to assess the taxonomic and phylogenetic status of species and populations. Fochetti et al. (2004) reported that the population from Balearic Islands showed high genetic distance values (allozyme data) from all other Tyrrhenoleuctra populations $(D=0.42-0.62) . D>0.16$ are the values usually displayed by stoneflies for separated species (Fochetti et al., 1997). The study also revealed fixed alleles at two of the 11 loci surveyed, suggesting it might be an independent taxon. Biochemical results were confirmed by mitochondrial DNA (mtDNA) data. Partial sequences of two mtDNA genes were amplified by polymerase chain reaction. A $1316 \mathrm{bp}$ long fragment of 